\title{
A Case Report of Clozapine-Induced Cardiomyopathy and Short Review of the Literature
}

\author{
Themistoklis Sertis*1, Dionysia Theocharidou ${ }^{2}$, Apostolos Theocharidis ${ }^{3}$ and Christos Chatz- \\ ieleftheriou ${ }^{4}$ \\ ${ }^{1}$ Cardiologist, Cardiology Department of Drama General Hospital, Drama, Greece
}

${ }^{2}$ Clinical Microbiologist, Laboratory of Hygiene and Environmental Protection, Medical School, Democritus University of Thrace, Greece

${ }^{3}$ Cardiologist, Laboratory of Hygiene and Environmental Protection, Medical School, Democritus University of Thrace, Greece

${ }^{4}$ Chief of Cardiology Department of Drama General Hospital, Drama, Greece

*Corresponding author: Themistoklis Sertis, Cardiologist, Cardiology Department of Drama General Hospital, Drama, Greece

\section{ARTICLE INFO}

Received: 慧 February 20, 2021

Published: 巣 March 25, 2021

Citation: Themistoklis Sertis, Dionysia Theocharidou, Apostolos Theocharidis, Christos Chatzieleftheriou. A Case Report of Clozapine-Induced Cardiomyopathy and Short Review of the Literature. Biomed J Sci \& Tech Res 34(5)-2021. BJSTR. MS.ID.005604

\section{ABSTRACT}

Clozapine is a very effective anti-psychotic agent accompanied by severe adverse effects such as neutropenia and cardiotoxicity. The present report is an attempt to describe a case of a patient with underlying heart disease, who developed clozapine-induced dilated cardiomyopathy and experienced clinical and echocardiographical improvement under supportive measures and drug discontinuation. The drug's cardiotoxic effect is furtherly discussed from a pathophysiological and therapeutic point of view.

Abbreviations: SGA: Second-Generation Antipsychotic Drug; EF: Ejection Fraction; MR: Mitral Regurgitation; MV: Mitral Valve; IVS: Intraventricular Septum; LV: Left Ventricle; LA: Left Atrium; IVC: Inferior Vena Cava; TR: Tricuspid Regurgitation; CBC: Complete Blood Count

\section{Introduction}

Ever since clozapine's introduction, as a second-generation antipsychotic drug (SGA), it is thought to be one of the most effective drugs against refractory schizophrenia. Clinical trials have identified its superiority against other SGAs presenting, overall, improved quality of life of first-line treatment-resistant schizophrenic patients. Among others, clozapine has been linked to better compliance, reduction of positive (delusions or hallucinations) and negative (such as lack of speech, depression, etc.) symptoms, and less suicidality [1]. Due to those beneficiary effects, based on different published evidence, it is recommended by many clinical practice guidelines as the gold standard to refractory schizophrenia. Although lacking extrapyramidal adverse effects, contrary to chlorpromazine, haloperidol, and other antipsychotic agents, it is accompanied by a wide range of severe or mild adverse effects including blood dyscrasia - neutropenia, cardiotoxicity, seizures, and sedation, hypotension, constipation, or metabolic syndrome which may be the cause of drug discontinuation [2,3].
It is of note that clozapine-associated cardiotoxicity requires close patient monitoring and can develop different manifestations including myocarditis, cardiomyopathy, and subclinical cardiac toxicity, caused by different pathophysiological and histological mechanisms.

\section{Aim}

The present study aims to present a case of a 65-year-old man under clozapine for the last 5 years, due to refractory schizophrenia and has been developing recurrent episodes of cardiac failure. Besides, pathophysiological and therapeutic measures of clozapinerelated cardiotoxicity are furtherly discussed, to unravel the way those patients should be managed.

\section{Case Report}

A 65-year-old male patient, who was under clozapine for the last five years, experienced repeated episodes of hospital admissions in the Cardiology Unit due to cardiac deterioration. 
The patient had experienced acute coronary syndrome two years ago, and underwent coronary angiography, presenting (LAD $100 \%$, RCA $100 \%$ ), which could not be treated surgically, based on cardiothoracic consultation. Heart failure was classified as NYHA III - IV (HFrEF), preserving an ejection fraction (EF) $=20-25 \%$. He also suffered from moderate to serious Mitral Regurgitation (MR). For those prementioned conditions he was under Furosemide 40 mg (gradual dose increase from $S: 1^{*} 1$ to $S: 1 * 2$ to $500 \mathrm{mg} \mathrm{S:} \mathrm{1/4*1),}$ Carvedilol 6,25mg S: $1 * 2$, Aspirine 100mg S: $1 * 1$, Eplerenone $25 \mathrm{mg}$ S: $1^{*} 1$, Ivabradine $5 \mathrm{mg} \mathrm{S:} \mathrm{1*2,} \mathrm{Atorvastatin} \mathrm{/} \mathrm{Ezetimibe} 10$ / $20 \mathrm{mg}$
S:1*1, Clozapine $200 \mathrm{mg} \mathrm{S:} 1 * 2$. On physical examination, the patient had rhythmic cardiac auscultation (S1, S2) and a systolic murmur of Mitral Valve (MV) $+3 / 6$. Pulmonary auscultation revealed diminished breath pneumonic sounds at the right lower and middle pulmonary lobe, crackles at the left lower lobe, and prolonged expiration. Upon abdomen palpation, a positive hepatojugular sign and substantial ascites were observed. The patient had lower extremities edema with indentation and vital signs BP $=100 / 60$ $\mathrm{mmHg}, \mathrm{HR}=95 / \mathrm{min}, \mathrm{SpO}_{2}=90 \%$.

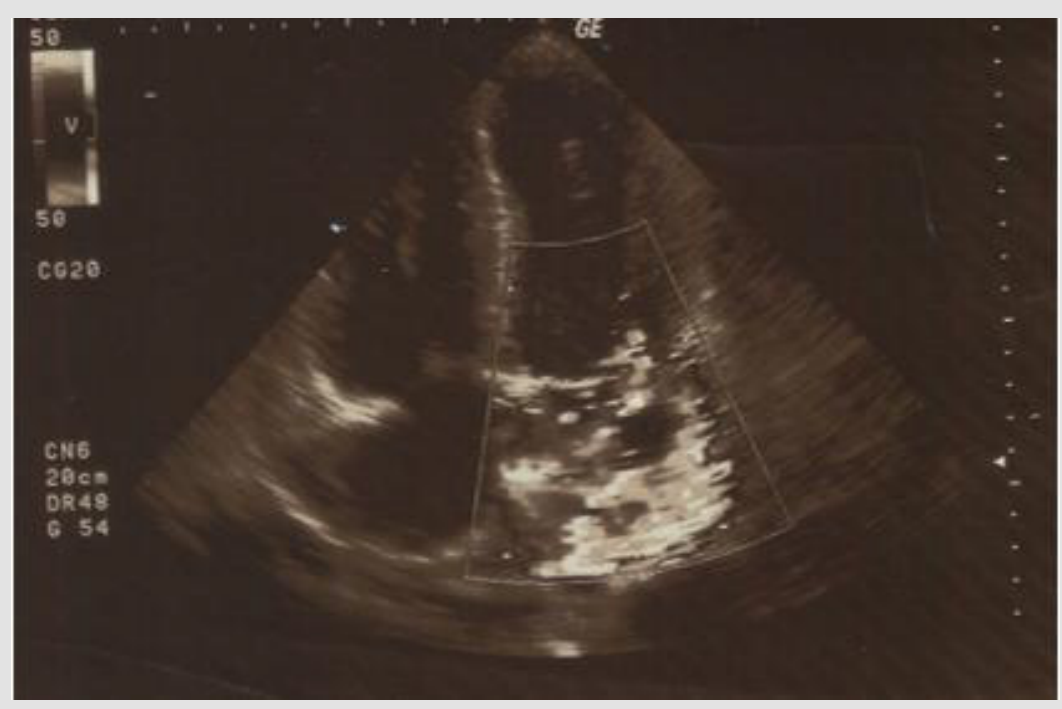

Figure 1: Four-chamber view: Mitral Regurgitation.

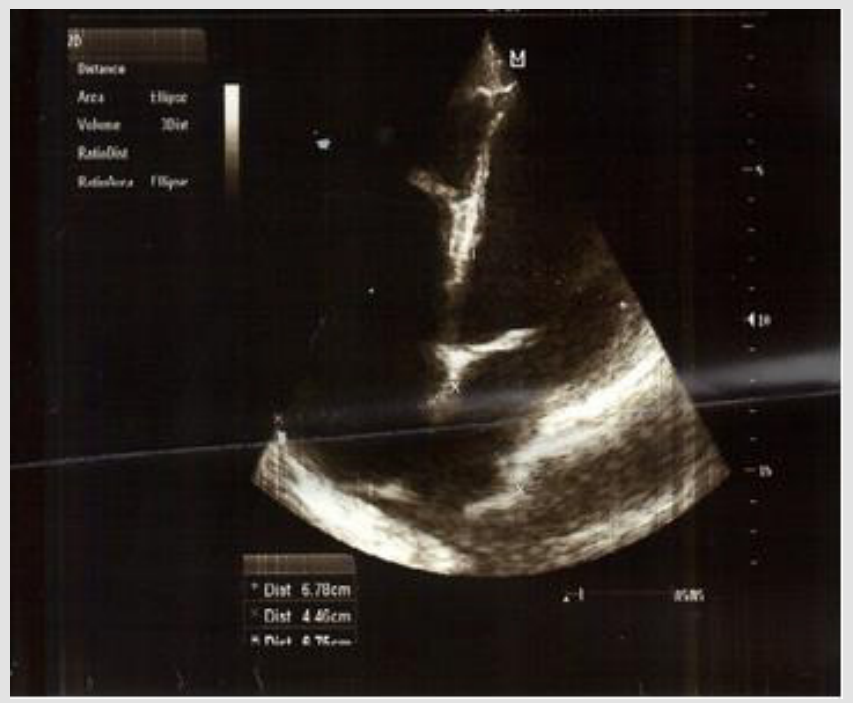

Figure 2: Four chamber view: LV dilation.

The ECG revealed SR tachycardia, with no signs of cardiac ischemia and repolarization abnormalities of the lateral cardiac wall. Echocardiography (shown in Figures 1 \& 2) revealed EF $=20-25 \%$, apical hypokinesia of left ventricle (LV), medial intraventricular septum (IVS) and lateral wall hypokinesia, LV enlargement: $68 \mathrm{~mm}$, medium left atrium (LA) enlargement: 48
$\mathrm{mm}(\mathrm{LA}$ area $=36 \mathrm{~cm} 2), \mathrm{MR}+2 / 4$, tricuspid regurgitation (TR) $+2 / 4$, inferior vena cava (IVC) $=22 \mathrm{~mm}$, with minimum breathing fluctuation during inhalation. Chest x-ray (shown in Figure 3) revealed cardiomegaly, congestion of lung hilum, reversion of pulmonary blood irrigation, and right pleural effusion. Upon admission, the patient was administered inotropic and diuretic 
treatment and underwent pleural and ascitic paracentesis several times. Biochemical and cytological examination of the paracentesis fluids didn't show signs of inflammation. After stabilization of the patient's clinical condition, he was admitted to a rehabilitation center, where the psychiatrist in charge ordered clozapine discontinuation and olanzapine commencement. The patient was followed by the Cardiology Unit as an outpatient and three months later, achieved an EF: 35- 40\%. Although the patient had a positive cardiac history, clozapine-induced dilated cardiomyopathy was diagnosed, a fact that was confirmed with the improved clinical condition of the patient when the drug's administration stopped.

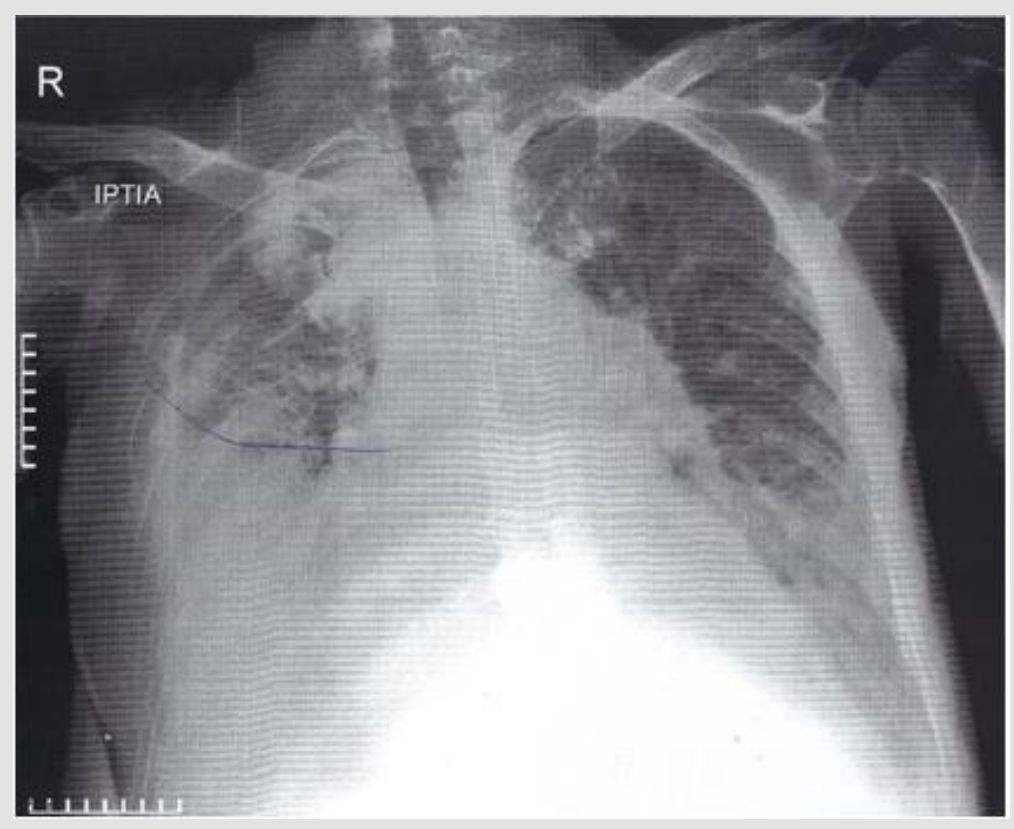

Figure 3: Chest x-ray: Pleural fluid (right) requiring paracentesis.

\section{Discussion}

As prementioned, clozapine cardiotoxicity may develop in different types of presentation such as myocarditis, dilated cardiomyopathy, or even subclinical cardiotoxicity, clinical conditions that are associated with $10 \%$ mortality rates $[3,4]$. Although there is no clinical evidence that those adverse effects are dose-related, they could be life-threatening, and require close clinical and laboratory monitoring, including troponin $\mathrm{T}$ or $\mathrm{I}$, CRP, and echocardiography [5]. Besides, the drug's prescription is recommended to be done with caution among patients with underlying heart disease and to be avoided among patients with severe underlying heart disease [4]. Further cardiological side effects are benign tachycardia (increase 10- 15 bpm on average), QT prolongation ( $>500 \mathrm{msec}$ ), and orthostatic hypotension.

Clozapine-induced myocarditis is an IgE mediated (hypersensitivity Type I) myocardium inflammation presenting with fever and signs of acute heart failure. Clinical symptomatology of the disease is unclear posing an impact in urgent and accurate diagnosis and treatment. Myocarditis takes place during the first two months of drug commencement and most cases are diagnosed during the first three weeks [6].

Therapeutic measures include supportive and preventive treatment to avoid cardiac overload and prevention of heart failure, whereas drug discontinuation is the key to the patient's clinical and biochemical improvement $[7,8]$. Mortality rates of clozapineinduced myocarditis can reach $21 \%$, a much higher rate than that observed among the general population [6]. Clozapine rechallenge is in general not recommended among those patients, although low dose and slow dose increase could be attempted, with the patient under close cardiology monitoring [3].

Clozapine-induced dilated cardiomyopathy presents with the main symptoms of cardiac failure (see the clinical presentation of our patient) and is diagnosed with echo findings, especially reduced EF and thin and dilated LV wall [3]. Although the exact pathophysiological mechanism of myocardial toxicity is unclear, the predominant path is IgE-mediated myocardial damage accompanied by eosinophilia or direct myocardial toxicity. This manifestation presents 4 weeks to years after the administration of the drug and is a rare adverse effect, affecting 1 among 1000 clozapine treated patients [4]. Apart from ECG and basic biochemical testing, NTproBNP provides further diagnostic information [4].Supportive treatment and patient stabilization are of note, accompanied by drug discontinuation. Most cases that have not experienced severe myocardial damage, achieve complete recovery after drug discontinuation and clozapine re- challenge could be attempted under close cardiology monitoring algorithm proposed by Cook, et al. [9]. Subclinical heart dysfunction presenting with no symptoms 
or mild tachycardia has been reported in $33 \%$ of young previously healthy clozapine patients [10]. The cause of this adverse effect has been linked to systematic inflammation, so as a result close cardiological, echocardiography, and biochemical monitoring of the patients are required both for prevention and treatment. B-blockers or ivabradine administration are indicated in these cases [11]. Last but not least, clozapine commencement may induce neutropenia or other forms of blood dyscrasia such as leukopenia or thrombopenia, especially during the first 18 weeks of drug administration. GSF can be used to stimulate granulocytes' production along with lithium administration. This condition may be severe, and potentially lifethreatening, however, drug discontinuation and the prementioned supportive measures can reverse the phenomenon [10].

\section{Conclusion}

$30 \%$ of psychotic patients fail to respond to first-line therapy. Clozapine is a very effective antipsychotic drug, which is under prescribed due to its severe side effects (blood dyscrasia and cardiotoxicity). To achieve proper adverse effect prevention or accurate diagnosis, from a cardiology point of view, a baseline cardiology consultation is required (including patient's history, echocardiography- EF, biochemical testing), before drug administration, and close contact between the psychiatrist in charge and the cardiologist, to identify and treat in time any adverse effect. From a hematological point of view, a complete blood count (CBC) is required before drug prescription, a test that should be repeated every week for the first 18 weeks.

\section{References}

1. S Subramanian, BA Völlm, N Huband (2017) Clozapine dose for schizophrenia. Cochrane Database of Systematic Reviews 2017(6): CD009555.

\section{ISSN: 2574-1241}

DOI: 10.26717/BJSTR.2021.34.005604

Themistoklis Sertis. Biomed J Sci \& Tech Res

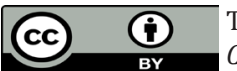

This work is licensed under Creative Commons Attribution 4.0 License

Submission Link: https://biomedres.us/submit-manuscript.php
2. S Warnez, S Alessi Severini (2014) Clozapine: A review of clinical practice guidelines and prescribing trends. BMC Psychiatry 14: 102.

3. G Kanniah, S Kumar (2020) Clozapine associated cardiotoxicity: Issues, challenges and way forward. Asian J Psychiatr 50: 101950

4. RK Patel, AM Moore, S Piper, M Sweeney, E Whiskey, et al. (2019) Clozapine and cardiotoxicity - A guide for psychiatrists written by cardiologists. Psychiatry Research 282: 112491.

5. K N Knoph, RJ Morgan, BA Palmer, KM Schak, AC Owen, et al. (2018) Clozapine-induced cardiomyopathy and myocarditis monitoring: A systematic review. Schizophr Res 199: 17-30.

6. BL Bellissima, MD Tingle, A Cicović, M Alawami, C Kenedi (2018) A systematic review of clozapine-induced myocarditis. Int J Cardiol 259: 122-129.

7. S Annamraju, B Sheitman, S Saik, A Stephenson (2007) Early recognition of clozapine-induced myocarditis. J Clin Psychopharmacol 27(5): 479483.

8. D De Berardis, N Serroni, D Campanella, L Olivieri, F Ferri, et al. (2012) Update on the Adverse Effects of Clozapine: Focus on Myocarditis. Curr Drug Saf 7(1): 55-62.

9. SC Cook, BA Ferguson, RO Cotes, TW Heinrich, AC Schwartz (2015) Clozapine-Induced Myocarditis: Prevention and Considerations in Rechallenge. Psychosomatics 56(6): 685-690.

10. C Rostagno, S Domenichetti, F Pastorelli, GF Gensini (2011) Usefulness of NT-Pro-BNP and Echocardiography in the Diagnosis of Subclinical Clozapine-Related Cardiotoxicity. J Clin Psychopharmacol 31(6): 712716.

11. J Lally, J Brook, T Dixon, F Gaughran, S Shergill, et al. (2014) Ivabradine, a novel treatment for clozapine- induced sinus tachycardia : a case series. Ther Adv Psychopharmacol Orig 4(3): 117-122.

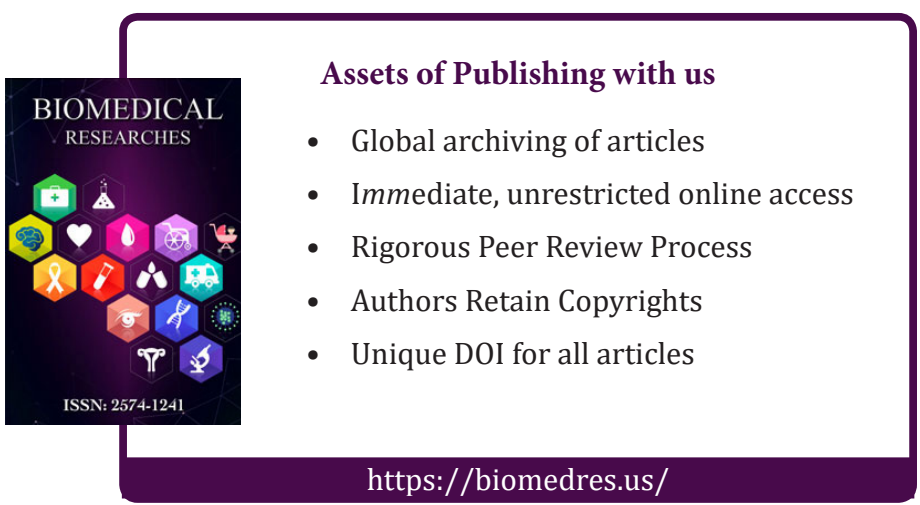

